\title{
Collagen remodelling in myocardia of patients with diabetes
}

\author{
M Shimizu, K Umeda, N Sugihara, H Yoshio, H Ino, R Takeda, Y Okada, I Nakanishi
}

\begin{abstract}
Aims: To investigate collagen remodelling in the interstitium of the heart in patients with diabetes.

Methods: Immunohistochemical study of the biopsied myocardium using type specific anticollagen antibodies (I, III, IV, V, VI) was performed in 12 patients with non-insulin dependent diabetes mellitus and six non-diabetic patients. There was no history of hypertension or coronary artery stenosis in any of the patients.

Results: Noticeable accumulations of collagen types I, III, and VI in the myocardial interstitium were recognised in both groups, but little accumulation of types IV or V was found. Types I and III mainly stained in the perimysium and perivascular region, while type VI predominantly stained in the endomysium. There was no disease specific accumulation of collagen in diabetes mellitus. The percentage of total interstitial fibrosis in the myocardium was significantly higher in the diabetic group than in the control group $(p<0 \cdot 05)$. Although the percentages of collagen types I and VI did not differ between the two groups, the percentage type of III was significantly higher in the diabetic group than in the controls (p $<0.01)$.
\end{abstract}

Conclusions: Collagen remodelling mainly as a result of an increase in collagen type III in the perimysium and perivascular region, occurs in the hearts of patients with diabetes.

(F Clin Pathol 1993;46:32-36)

Second Department of Internal Medicine

M Shimizu

K Umeda

N Sugihara

H Yoshio

$H$ Ino

R Takeda

First Department of

Pathology, School of

Medicine, Kanazawa

University, Japan

Y Okada

I Nakanishi

Correspondence to:

Dr Masami Shimizu, The

Second Department of

Internal Medicine, School of

Medicine, Kanazawa

University, Takara-machi

13-1, Kanazawa 920, Japan

Accepted for publication

7 August 1992
Primary myocardial injury has been shown in diabetes mellitus even in the absence of hypertension and coronary atherosclerosis. ${ }^{12}$ The histopathological changes found in the myocardium of patients with diabetes include myocyte hypertrophy, interstitial fibrosis, and perivascular fibrosis. Interstitial fibrosis is composed largely of fibrillar collagen. Regan $e t$ al reported the presence of interstitial collagen deposition in left ventricular biopsy specimens in diabetic patients, and found collagen accumulation in perivascular loci, between myofibres, or as replacement fibrosis in a postmortem study of cardiac tissue of diabetics. ${ }^{3}$ On the other hand, it has been reported that many phenotypes with differing molecular structures are present in the fibrillar collagen, with the distribution of these pheno- types varying in different tissues. ${ }^{45}$ Weber et al carried out several studies on myocardial collagen remodelling. ${ }^{6-8}$ However, no information is available on the changes in collagen in the myocardium of patients with diabetes. Accordingly, we investigated the localisation of collagen phenotypes in the interstitium of such cardiac tissue and estimated them quantitatively using an immunohistochemical method.

\section{Methods}

Biopsy specimens were obtained from 12 patients with non-insulin dependent diabetes mellitus who had undergone cardiac catheterisation because of complaints of chest pain or electrocardiographic abnormalities. In all patients the absence of more than $25 \%$ coronary artery stenosis was documented angiographically, and there was no history of hypertension, chronic renal failure, or other endocrinological disorders. Biopsy specimens from six non-diabetic patients, in whom cardiac catheterisation including endomyocardial biopsy was performed as. part of the evaluation of arrhythmia or chest symptoms, but in whom no clinically relevant coronary artery stenosis or abnormal haemodynamics was found, served as controls. Diabetes mellitus was diagnosed and classified in accordance with the criteria of the National Diabetes Data Group. ${ }^{9}$ The diabetic patients comprised six men and six women (mean (range) age 52.1 (2.9) years). The controls comprised three men and three women with a mean age of $43.5(3.7)$ years. The duration of diabetes mellitus ranged from one to 17 years (mean 10.5 years). The degree of diabetic complications ranged from mild to moderate. A right ventricular endomyocardial biopsy was performed using the Konno-Sakakibara bioptome or Bipal biopsy forceps (Cordis), and specimens were obtained from the region of the interventricular septum. Informed consent was obtained from each patient before cardiac catheterisation.

\section{HISTOPATHOLOGICAL STUDY}

The specimens obtained by endomyocardial biopsy were submerged immediately in $10 \%$ buffered formalin. After being embedded in paraffin wax they were cut into $4 \mu \mathrm{m}$ sections on a longitudinal plane and more than eight continuous sections were obtained from each specimen. One of these sections was stained with haematoxylin and eosin, another was stained with Mallory-Azan; the other sections were stained with type specific anti-collagen 
antibodies or normal rat $\operatorname{IgG}$ as a negative control.

IMMUNOHISTOCHEMICAL STUDY

Sections of paraffin wax embedded myocardium were treated with $0.05 \%$.protease type XXIV (Sigma, USA) for 30 minutes; nonspecific reactions were checked for with $0.3 \%$ hydrogen peroxide. The prepared sections were stained by the avidin-biotin-complex (ABC) method $^{10}$ using type specific anticollagen antibodies (types I, III, IV, V, VI) which had been purified. $^{11}$

\section{LOCALISATION OF COLLAGEN TYPES}

Myocardial fibrosis in the sections stained with Mallory-Azan was classified into three components: (1) endomysial fibrosis-fibrosis providing lateral cell to cell connections; (2) perimysial fibrosis-fibrosis surrounding myocytes to form muscle bundles; (3) perivascular fibrosis-fibrosis surrounding intramyocardial small vessels $10 \mu \mathrm{m}-50 \mu \mathrm{m}$ in diameter. We used the stain of the endocardium to identify the potential effects on staining quality caused by fixation of the specimen, density of the antibodies, and other technical problems. The distribution of each type of collagen in each region was assessed by three cardiac pathologists, based on the following system: $(-)$ no staining; $(1+)$ only slight staining; $(2+)$ somewhat intense staining, but less intense than that of the endocardium; $(3+)$ intense staining equivalent to that of the endocardium. Blood vessels $10-50 \mu \mathrm{m}$ were found in biopsy specimens obtained from nine (two controls and seven diabetic patients) out of the 18 subjects, with perivascular fibrosis evaluated in these specimens.
QUANTIFICATION OF TOTAL MYOCARDIAL INTERSTITIAL FIBROSIS AND VARIOUS TYPES OF COLLAGEN

The extent of total fibrosis and various types of collagen was measured by the point counting method $^{1213}$ using the sections stained with Mallory-Azan and each type specific anticollagen antibody. We used a point counting method grid with the points arranged in a square pattern and a distance of $10 \mu \mathrm{m}$ between points. More than 2000 points, which lay on the myocardial cells or on the fibrotic tissue stained blue by Mallory-Azan and brown by type specific anticollagen antibodies only in the longitudinally cut cells, were recognised. The percentage of fibrosis was calculated according to the formula:

percentage of fibrosis $=$ points lying on the fibrosis

points lying on the myocytes and fibrosis $\times 100$.

All data were expressed as mean (1 SEM). Statistical analysis was performed using Student's unpaired $t$ test and the Mann-Whitney $U$ test. Differences were considered statistically significant when the $p$ value was less thill $0 \cdot 05$.

\section{Results}

Substantial accumulations of collagen types I, III, VI in the myocardial interstitium were recognised in both groups (figs 1-3). On the other hand, no accumulation of collagen types IV or V was found in either group, except for a slight accumulation of collagen type IV in the intima of the small vessels. Collagen type I was mainly stained in the perimysium and perivas-

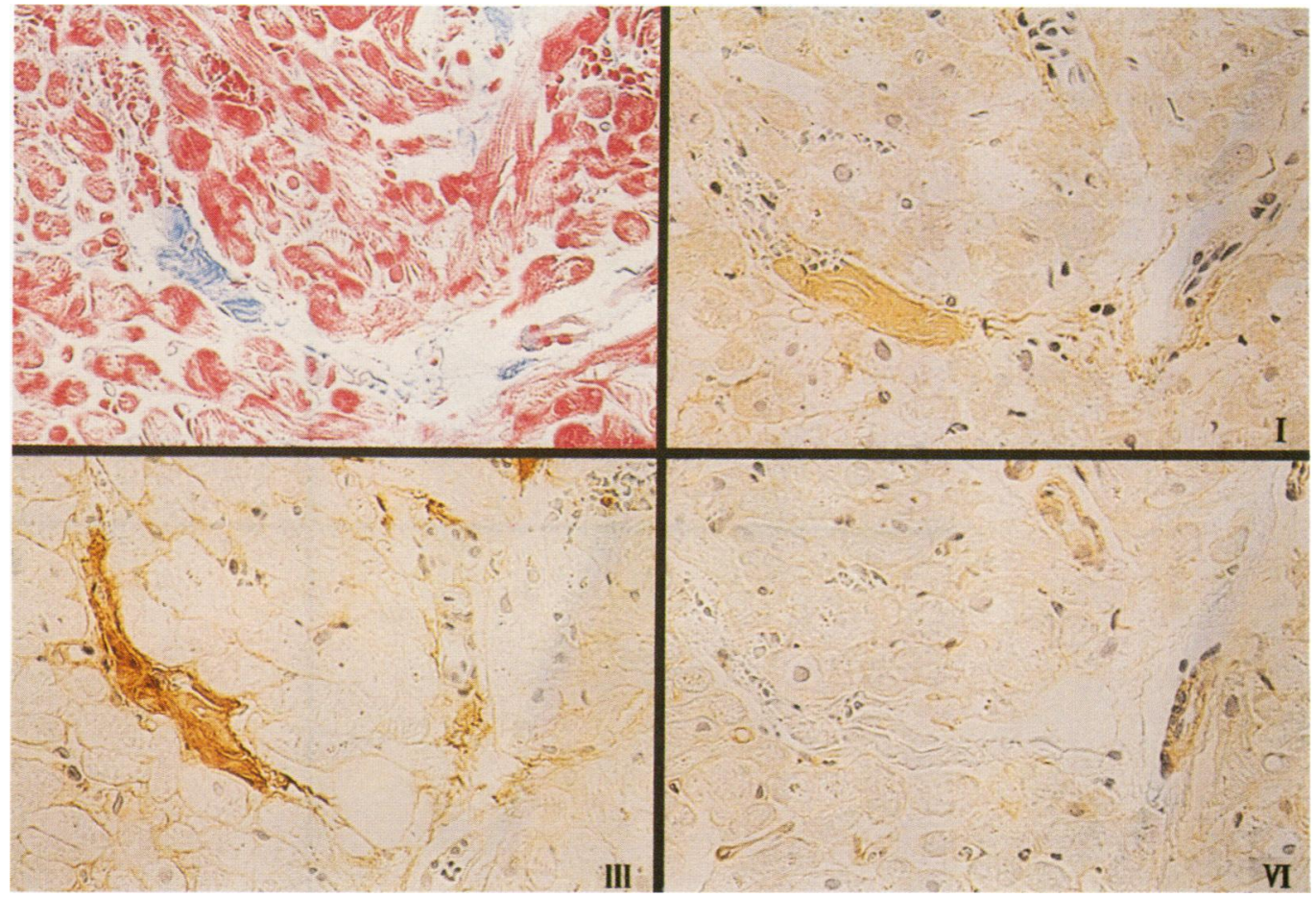

Figure 1 Perimysial fibrosis stained with Mallory-Azan (upper left) and immunostained with antibodies to type I (upper right), type III (lower left) and type VI (lower right) collagens. 
Figure 2 Endomysial fibrosis stained with Mallory-Azan (upper lefi) and immunostained with antibodies to type I (upper right), type III (lower left) and type VI (lower right) collagens.
Figure 3 Perivascular fibrosis stained with Mallory-Azan (upper left) and immunostained with antibodies to type I (upper right), type III (lower left) and type VI (lower right) collagen.

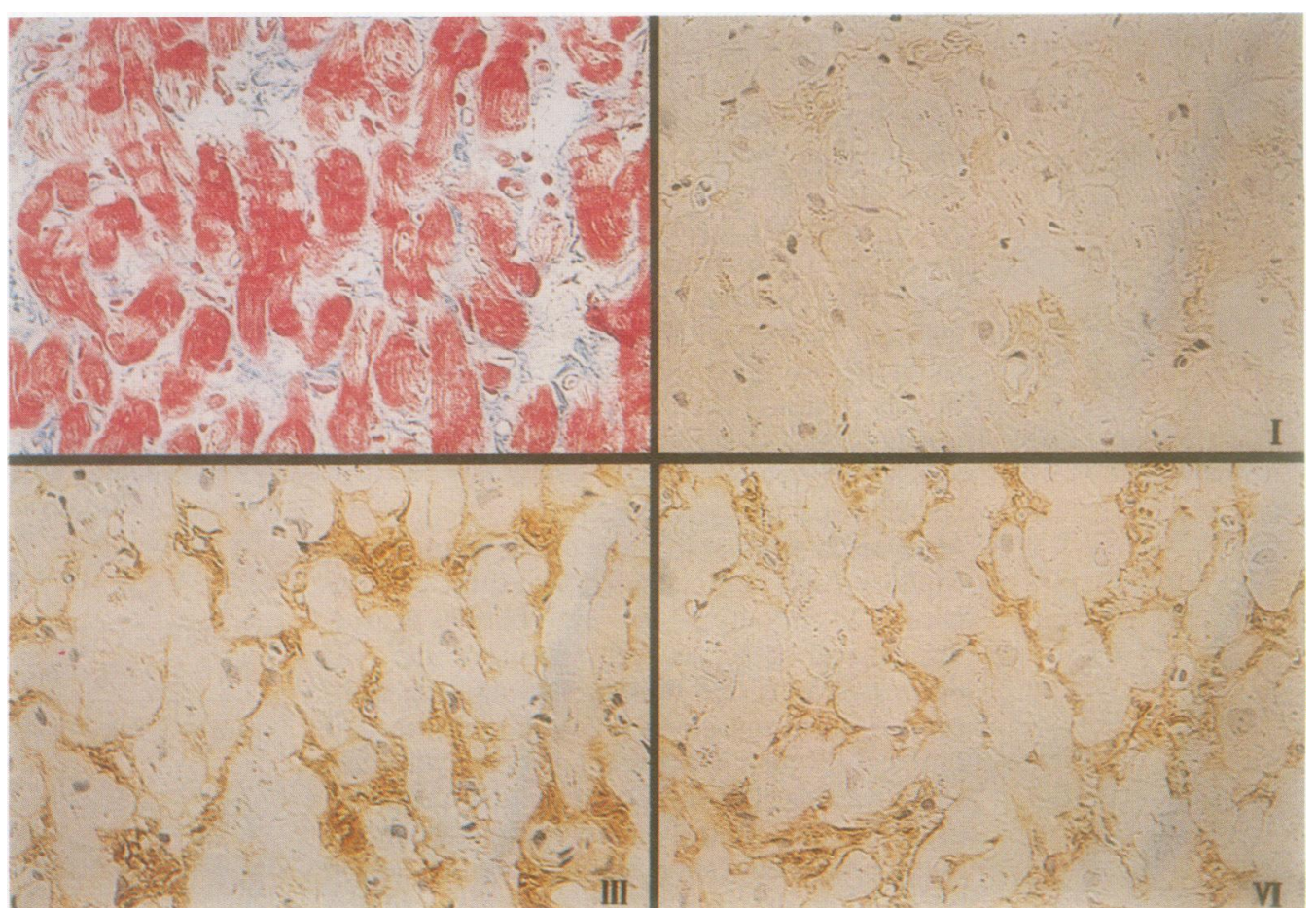

cular region, but only slightly stained in the endomysium. Collagen type III was intensely stained in the perimysium and perivascular region and weakly stained in the endomysium. Collagen type VI was intensely stained in the endomysium and weakly stained in the perimysium and perivascular region (fig 4). There was no disease specific accumulation of collagen in diabetes mellitus.

The percentage of fibrosis in the sections stained with Mallory-Azan was significantly higher in the diabetic group than in the control group $(17.0(2.8)$ v $7.2(1.9) \% ; \mathrm{p}<0.05)$. Figure 5 shows a comparison of the percentage of collagen types I, III, VI in the two groups. The percentages of collagen types I and VI did

not differ significantly between the two groups (type I:diabetes mellitus 11.4 (3.0); controls 4.3 (1.3)\%; type VI:diabetes mellitus 24.5 $(2.5)$; controls $22.0(3.4) \%)$. On the other hand, the percentage of collagen type III in diabetes mellitus was significantly higher than that of the controls $(19.6(2.3) v 7.8(3.3) \%$; $\mathrm{p}<0.01)$.

\section{Discussion}

Interstitial fibrosis was significantly increased in the group with diabetes mellitus. Most of this was composed of collagen fibres. Several types of differing molecular structures ${ }^{45}$ are present in these collagen fibres, but it is not

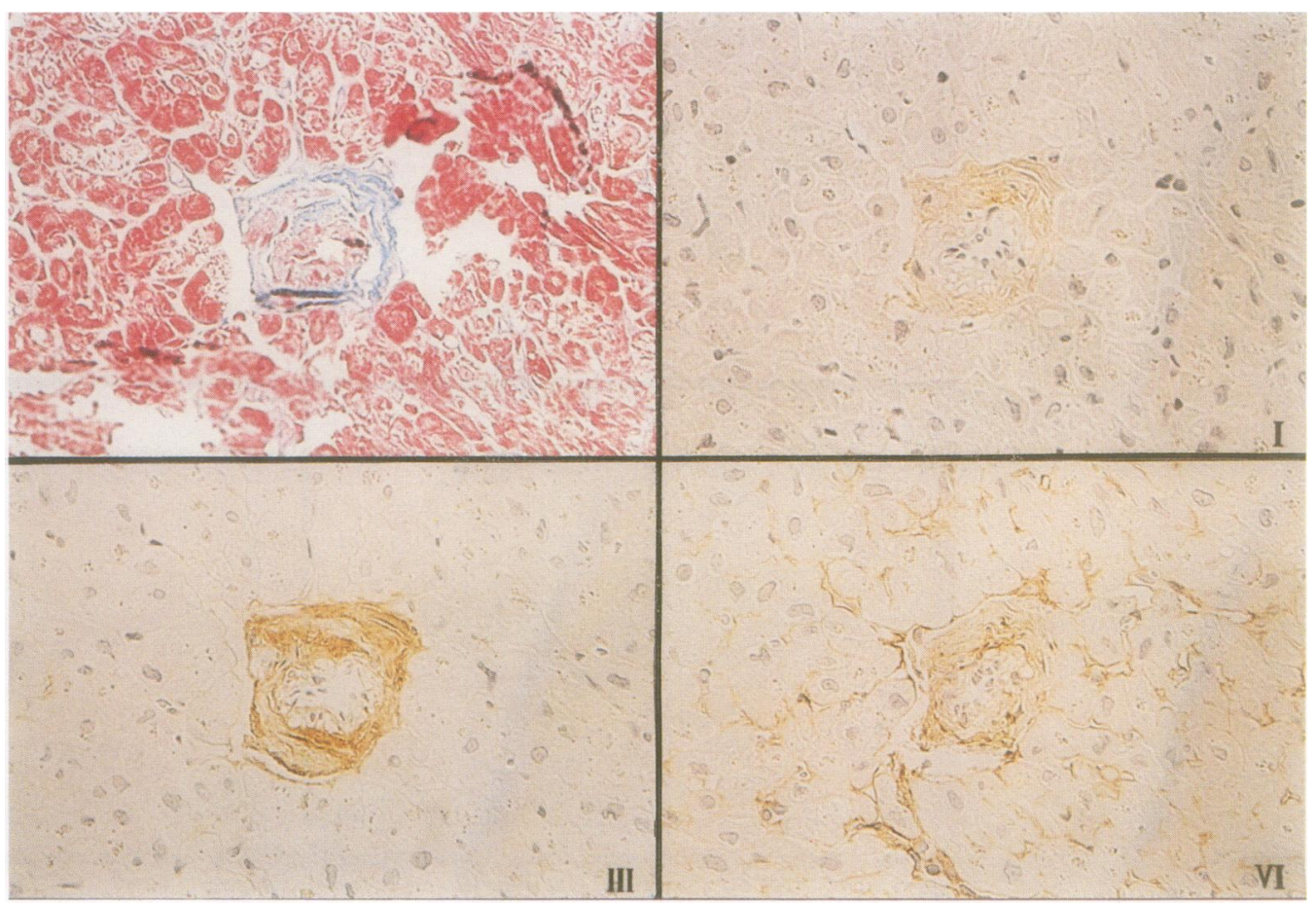


Type I

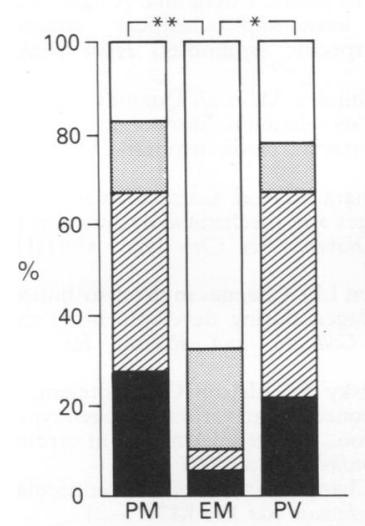

Type III

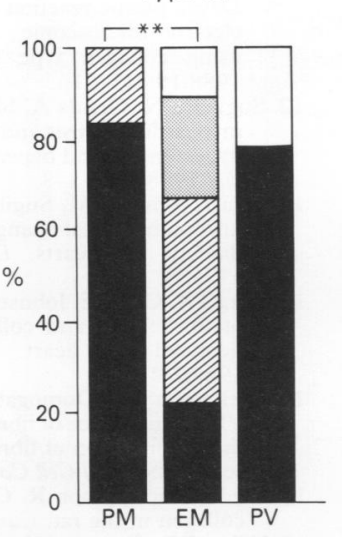

$:(3+) \square:(1+)$
Figure 4 Semiquantitative analysis of the distribution of collagen types I, III and VI. $P M=$ perimysium, $E M=$ endomysium, $P V=$ perivascular region. Staining pattern: $(-)$ not detectable, $(1+)$ trace, $(2+)$ moderate, $(3+)$ abundant.

Figure 5 Comparison of the percentages of collagen types I, III and VI in the two groups. The percentage of collagen type III in diabetes mellitus was significantly higher than that of the controls.
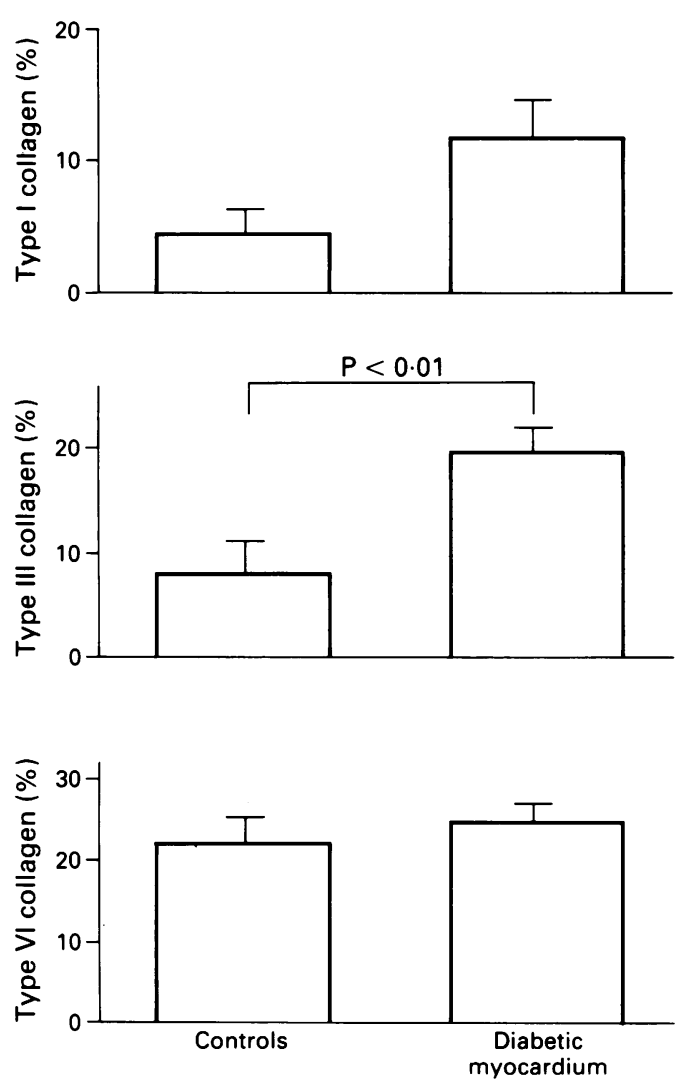

clear whether differences in the localisation or proportion of these collagen types exist in the hearts of patients with diabetes.

Differences were found in the distribution of each type of collagen among tissues, although this distribution was similar in both groups. Collagen types I and III are representative of interstitial fibrosis. Borg et $\mathrm{al}^{14}$ and Shekhonin et $a l^{15}$ reported results similar to those obtained in the present study, suggesting that the localisation of collagen types I and III in the diabetic heart is not specific for this disease.

No differences in the amounts of types I or
VI were found between the diabetic and control groups, but type III was significantly increased in patients with diabetes. The mechanism involved is unknown, but myocardial ischaemia is presumed to have a role. In the hearts of patients with diabetes luminal narrowing due to intramural coronary arteriole wall thickening or deposition of periodic acid Schiff positive material and perivascular fibrosis are present. As a result the diabetic myocardium is exposed to ischaemia. As Medugorac et $a^{16}$ speculated about hypertrophied cardiac tissue, the diminished blood flow in the diabetic myocardium over extended periods may lead to myocyte necrosis and subsequent healing, resulting in increased synthesis of type III collagen by cardiac fibroblasts. On the other hand, Villee et $a l,{ }^{17}$ in experiments using human skin fibroblasts cultured in vitro, found an increased glucose concentration in the medium and increased production of collagen type III. This interesting report suggests that in diabetes mellitus, in addition to the development of ischaemia due to microangiopathy, the increased glucose concentration may itself influence collagen metabolism. Due to the increase in blood glucose, fibrillar collagen begins to accumulate in the adventitia of intramural coronary arteries and then extends into the intramuscular spaces; this is followed by an abnormality in the permeability of these intramural vessels. ${ }^{18} \mathrm{An}$ increase in coronary vascular permeability may also facilitate the influx of growth factors in the interstitium and lead to further fibroblast proliferation and enhanced collagen synthesis. Type III collagen in myocardial interstitium may then be increased by the above mentioned mechanisms. In addition to cardiac fibroblast proliferation or enhanced collagen synthesis, collagen degradation may be retarded, although no information is available about this. Other interesting questions include which factors determine collagen synthesis in the cardiac tissues of patients with diabetes (for example, in addition to angiotensin II and aldosterone reported in the pressure overloaded left ventricle, ${ }^{1819}$ the involvement of glucose, insulin, etc.) and what kind of drug might inhibit this process. It is expected that ongoing studies using molecular biological techniques will shed light on these issues.

Collagen is the most important of the extracellular structural proteins, with collagen fibres acting as lateral connections between cells and muscle bundles to govern architecture, maintain shape and wall thickness, and resist myocardial deformation. However, while collagen is the major determinant of myocardial stiffness, ${ }^{20}{ }^{21}$ collagen phenotype remodelling may also influence cardiac function. Bouchard et $a l^{22}$ in an echocardiographic study, reported a reduced left ventricular enddiastolic volume index and impaired left ventricular diastolic filling in diabetics, ${ }^{23}$ and Regan et $a^{3}$ also reporting increased left ventricular chamber stiffness. We surmise that the increase in interstitial fibrosis and alterations in collagen phenotypes found in the present study may in some way be related to 
these changes in cardiac function, although further detailed investigations will be needed to clarify this.

1 Rubler S, Dlugash J, Yuceoglu YZ, Kumral T, Branwood AW, Grishman A. New type of cardiomyopathy associated with diabetic glomerulosclerosis. Am $\mathscr{f}$ Cardiol 1972 with diabetic

2 Ledet T. Diabetic cardiomyopathy. Quantitative histological studies of the heart from young juvenile diabetics. Acta Pathol Microbiol Scand (Sect A) 1976;84:421-8.

3 Regan TJ, Lyons MM, Ahmed SS, et al. Evidence for cardiomyopathy in familial diabetes mellitus. $\mathcal{f}$ Clin Inves 1977;60:885-99.

4 Miller EJ. The structure of fibril-forming collagens. Ann NY Acad Sci 1985;460:1-13.

5 Kuhn K. The collagens-molecular and macromolecular structures. In: Tschesche $\mathrm{H}$, ed. Proteinases in inflammation and tumour invasion. Berlin: Walter de Gruvter \& Co. 1986:107-43.

6 Weber KT, Janicki JS, Shroff SG, Pick R, Chen RM, Bashey RI. Collagen remodelling of the pressure-overloaded hypertrophied nonhuman primate myocardium. Circ Res 1988;62:757-65.

7 Jalil JE, Doering CW, Janicki JS, Pick R, Shroff SG, Weber $\mathrm{KT}$. Fibrillar collagen and myocardial stiffness in the intact hypertrophied rat left ventricle. Circ Res 1989 64:1041-50.

8 Weber KT, Pick R, Silver MA, et al. Fibrillar collagen and remodelling of dilated canine left ventricle. Circulation 1990;82:1387-401.

9 National Diabetes Data Group. Classification and diagnosis of diabetes mellitus and other categories of glucose intolerance. Diabetes 1979;28:1039-57.

$10 \mathrm{Hsu}$ S, Raine L, Fanger H. A comparative study of the peroxidase-anti-peroxidase method and an avidin-biotin complex method for studying polypeptide hormones with radioimmunoassay antibodies. Am $¥$ Clin Pathol 1981; 75:734-8.
11 Minamoto T, Ooi A, Okada Y, Mai M, NagaiY, Nakanishi I. Desmoplastic reaction of gastric carcinoma: A light- and electron-microscopic immunohistochemical analysis using collagen type specific antibodies. Hum Pathol 1988;19:815-21

12 Sugihara N, Genda A, Shimizu M, et al. Quantification of myocardial fibrosis and its relation to function in essential hypertension and hypertrophic cardiomyopathy. Clin Cardiol 1988;11:771-8.

13 Kita Y, Shimizu M, Sugihara N, et al. Correlation between histopathological changes and mechanical dysfunction in distopathological changes and mechanical dysfunction in diabetic

14 Borg TK, Gay RE, Johnson LD. Changes in the distribution of fibronectin and collagen during development of the
neonatal rat heart. Collagen and Related Research neonatal rat
$1982 ; 2: 211-8$.

15 Shekhonin BV, Domogatsky SP, Idelson GL, Koteliansky VE. Participance of fibronectin and various collagen types in the formation of fibrous extracellular matrix in cardiosclerosis. $\mathcal{F} \mathrm{Mol}$ Cell Cardiol 1988;20:501-8.

16 Medugorac I, Jacob R. Characterisation of left ventricular collagen in the rat. Cardiovasc Res 1983;17:15-21.

17 Villee DB, Powers ML. Effect of glucose and insulin on collagen secretion by human skin fibroblasts in vitro. Nature 1977;268:156-8.

18 Weber KT, Brilla CG. Pathological hypertrophy and cardiac interstitium. Fibrosis and renin-angiotensin-aldosterone system. Circulation 1991;83:1849-65.

19 Brilla CG, Pick R, Tan LB, Janicki JS, Weber KT Remodelling of the rat right and left ventricles in Remodelling of the rat right and left ventricles in

20 Borg TK, Ranson WF, Moslehy FA, Caulfield JB. Structura basis of ventricular stiffness. Lab Invest 1981;44:49-54.

21 Borg TK, Caulfield JB. The collagen matrix of the heart. Fed Proc 1981;40:2037-41.

22 Bouchard A, Sanz N, Botvinick EH, et al. Non-invasive assessment of cardiomyopathy in normotensive diabetic patients between 20 and 50 years old. $A m, \mathcal{J} M e d$ 1989;87:160-6.

23 Shimizu M, Sugihara N, Kita Y, Shimizu K, Shibayama S, Takeda $R$. Increase in left ventricular chamber stiffness in patients with non-insulin dependent diabetes mellitus. patients with non-insulin
fpn Circ 7 1991;55:657-64. 